\title{
The Validity and Sensitivity of Rheumatoid Arthritis Pain Scale on a Different Ethnic Group From Indian Rheumatoid Arthritis Patients
}

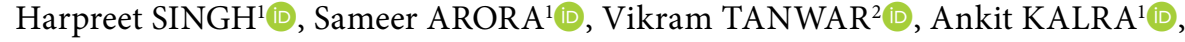 \\ Gagandeep SUKHIJA ${ }^{1}$, Nikhil GOVIL ${ }^{3}$ (D) \\ ${ }^{1}$ Department of Rheumatology, Pt. B. D. S. Post Graduate Institute of Medical Sciences, Rohtak, India \\ ${ }^{2}$ Department of General Medicine, S H K M Government Medical College, Nalhar, India \\ ${ }^{3}$ Department of General Medicine, K C G M C, Karnal, India
}

\begin{abstract}
Objectives: This study aims to assess pain in rheumatoid arthritis (RA) patients by using Rheumatoid Arthritis Pain Scale (RAPS) and to find its correlation with Disease Activity Score 28 (DAS28) and Clinical Disease Activity Index (CDAI).

Patients and methods: The study included 100 RA patients (23 males, 77 females; mean age 43.22 years; range, 19 to 72 years) who were subjected to RAPS questionnaire for pain assessment and DAS28 and CDAI for disease activity assessment. Spearman's correlation coefficient was measured to assess the correlation of RAPS with DAS28 and CDAI. Cronbach's alpha (a) was also measured for each scale to assess reliability.

Results: The study group had a female to male ratio of 3.34:1. Mean values for RAPS, DAS28 and CDAI were 62.91, 5.59, and 25.24, respectively. RAPS was correlated with DAS28 and CDAI with correlation coefficients of 0.811 and 0.770 , respectively. Cronbach's a for RAPS, DAS28 and CDAI were $0.892,0.814$, and 0.833 , respectively.

Conclusion: Rheumatoid Arthritis Pain Scale had a strong positive correlation with disease activity measures of DAS28 and CDAI. RAPS also showed good correlation with core data set measures hence merits its place in clinical practice.

Keywords: Clinical Disease Activity Index, Cronbach's alpha, disease activity score 28, Rheumatoid Arthritis Pain Scale, Spearman's correlation coefficient.
\end{abstract}

Rheumatoid arthritis (RA) is a chronic, multisystemic, immune-mediated inflammatory disease characterized by articular and extraarticular manifestations along with systemic manifestations in the form of generalized malaise and fatigue. ${ }^{1}$ It exhibits a chronic fluctuating course, which may result in recurrence of symptoms, progressive joint damage, deformity and disability. ${ }^{1}$ Typical symptoms of RA are multiple joint pain, early morning stiffness and joint swelling, but out of these, pain is the most common reason for seeking medical care as observed in various studies. ${ }^{2-4}$ Joint pain in RA is diffuse aching and burning that is usually moderately severe, and usually intermittent with exacerbations and remissions. ${ }^{5}$ Pain is the outcome of its four components, i.e. physiological, affective, sensorydiscriminative, and cognitive. ${ }^{5}$ RA patients mainly present in clinics or outpatient departments with the complaint of pain, which may be mild to severe in intensity. Rheumatoid Arthritis Pain Scale (RAPS) is a self-reported pain assessment scale, which is easy to use and which assesses almost all aspects of RA pain. ${ }^{5}$ Only limited

Received: December 09, 2018 Accepted: April 22, 2019 Published online: November 06, 2019

Correspondence: Vikram Tanwar, MD. Department of General Medicine, S H K M Government Medical College, 122107 Nalhar, India. Tel: 9050011361 e-mail:drvikrampgi@gmail.com 
data are available on RAPS use in RA patients and to our knowledge, no Indian study has been performed to date. Therefore, in this study, we aimed to assess pain in RA patients by using RAPS and to find its correlation with Disease Activity Score 28 (DAS28) and Clinical Disease Activity Index (CDAI).

\section{PATIENTS AND METHODS}

The study was conducted at Department of Rheumatology, Pt. B. D. S. Post Graduate Institute of Medical Sciences between July 2014 to April 2015 and included 100 RA patients (23 males, 77 females; mean age 43.22 years; range, 19 to 72 years) diagnosed as per American College of Rheumatology (ACR) 1987 criteria $^{6}$ and who were able to read and write. Patients who were severely anemic, had hypothyroidism or evidence of liver, renal, pulmonary or cardiac disease were excluded. The study protocol was approved by the institutional Ethics Committee. A written informed consent was obtained from each patient. The study was conducted in accordance with the principles of the Declaration of Helsinki.

All patients were assessed for both patientand physician-derived parameters. Patientderived parameters were pain visual analog scale (VAS) and patient's global assessment (PGA) of disease activity whereas physicianderived parameters were tender joint count (TJC), swollen joint count (SJC) and evaluator's global assessment (EGA) of disease activity. Erythrocyte sedimentation rate (ESR) was also measured for each patient. Disease activity measures of DAS28 and CDAI were calculated by using these baseline parameters.

All patients were asked to complete the RAPS questionnaire simultaneously and to score each question from 0 (never) to 6 (always). Total RAPS score was calculated by simple summation of individual item's score ranging from 0 to 6 totaling between 0 and 144 with higher scores indicating more pain. There is no cut-off limit to define mild, moderate and severe pain in RAPS score. ${ }^{5}$

\section{Statistical analysis}

Data were statistically analyzed using IBM SPSS version 20.0 software (IBM Corp., Armonk,
NY, USA). Spearman's correlation coefficient was calculated to interpret relationships between two variables. The strength of correlation as per norms was classified into nil (zero), fair (0.1-0.25), moderate $(0.25-0.50)$, strong $(0.5-0.8)$, and very strong to perfect $(0.8-1)$. $\mathrm{P}$ values of less than 0.05 was considered as statistically significant. All patients were distributed into four groups as per quantification of disease severity assessed by

Table 1. Mean values with standard deviation of various parameters

\begin{tabular}{lc}
\hline Parameters/variable & Mean \pm SD \\
\hline TJC (0-28) & $8.8 \pm 5.7$ \\
SJC (0-28) & $6.3 \pm 3.9$ \\
PGA (0-100 mm) & $57 . \pm 18.8$ \\
EGA (0-100 mm) & $49.0 \pm 17.5$ \\
Pain VAS (0-100 mm) & $5.7 \pm 1.9$ \\
ESR (0-200 mm/hour) & $43.8 \pm 20.0$ \\
DAS28 (0-9.4) & $5.6 \pm 1.2$ \\
CDAI (0-76) & $25.2 \pm 11.2$ \\
RAPS (0-144) & $62.9 \pm 22.0$ \\
\hline SD: Standard deviation; TJC: Tender joint count; SJC: Swollen joint count; \\
PGA: Patient's global assessment; EGA: Evaluator's global assessment; VAS: \\
Visual analog scale; ESR: Erythrocyte sedimentation rate; DAS28: Disease \\
activity score 28; CDAI: Clinical Disease Activity Index; RAPS: Rheumatoid \\
Arthritis Pain Scale.
\end{tabular}

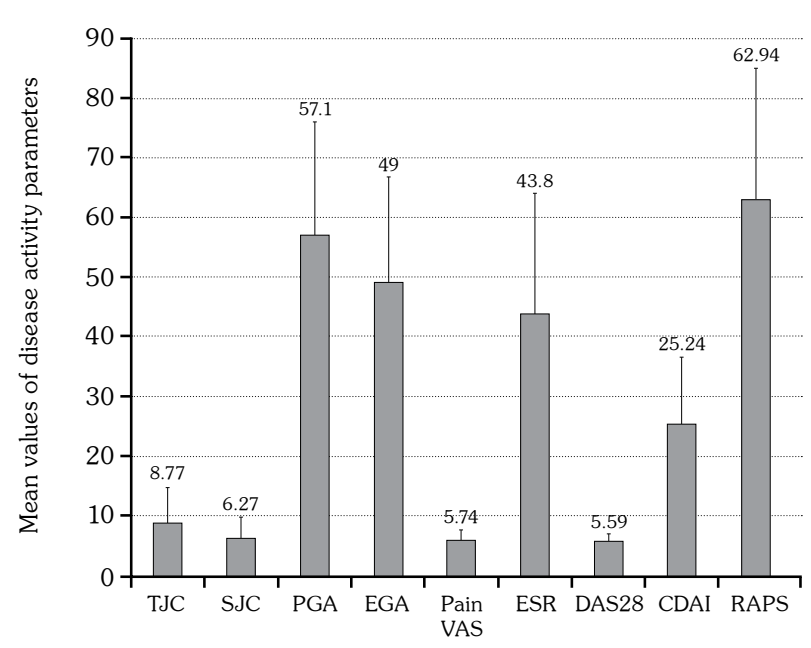

Figure 1. Column chart showing values within one standard deviation of mean for various disease activity parameters.

TJC: Tender joint count; SJC: Swollen joint count; PGA: Patient's global assessment; EGA: Evaluator's global assessment; VAS: Visual analog scale; ESR: Erythrocyte sedimentation rate; DAS28: Disease activity score 28; CDAI: Clinical Disease Activity Index; RAPS: Rheumatoid Arthritis Pain Scale. 
Table 2. Correlation of RAPS with DAS28

\begin{tabular}{|c|c|c|c|c|c|}
\hline \multirow[t]{2}{*}{ Severity } & \multirow{2}{*}{$\begin{array}{c}\text { Number of patients } \\
n\end{array}$} & DAS28 & RAPS & $\mathrm{CC}$ & \multirow[b]{2}{*}{$p$} \\
\hline & & Mean \pm SD & Mean \pm SD & $p$ & \\
\hline Remission & 1 & $2.5 \pm 0.0$ & $21 \pm 0.0$ & & \\
\hline Low & 3 & $3.0 \pm 0.1$ & $23.3 \pm 6.0$ & 0.894 & $<0.001$ \\
\hline Moderate & 31 & $4.4 \pm 0.6$ & $45.5 \pm 13.8$ & 0.660 & $<0.001$ \\
\hline High & 65 & $6.3 \pm 0.7$ & $73.7 \pm 17.1$ & 0.585 & $<0.001$ \\
\hline Overall & 100 & $5.6 \pm 1.2$ & $62.9 \pm 2.0$ & 0.811 & $<0.001$ \\
\hline
\end{tabular}

RAPS: Rheumatoid Arthritis Pain Scale; DAS28: Disease activity score 28; CC: Correlation coefficient; SD: Standard deviation; Disease severity as per disease activity score 28: remission=0-2.6; mild=2.61-3.2; moderate=3.21-5.1; severe=5.11-9.4; $<<0.05$ : Significant.

Table 3. Correlation of RAPS with CDAI

\begin{tabular}{|c|c|c|c|c|c|}
\hline \multirow[t]{2}{*}{ Severity } & \multirow{2}{*}{$\begin{array}{c}\text { Number of patients } \\
n\end{array}$} & CDAI & RAPS & $\mathrm{CC}$ & \multirow[b]{2}{*}{$p$} \\
\hline & & Mean \pm SD & Mean \pm SD & $p$ & \\
\hline Low & 13 & $7.9 \pm 2.2$ & $34.4 \pm 16.8$ & -0.09 & $>0.05$ \\
\hline Moderate & 28 & $18.1 \pm 3.0$ & $51.8 \pm 15.4$ & 0.46 & $<0.05$ \\
\hline High & 59 & $32.5 \pm 8.0$ & $74.5 \pm 16.7$ & 0.62 & $<0.05$ \\
\hline Overall & 100 & $25.2 \pm 11.2$ & $62.9 \pm 22.0$ & 0.770 & $<0.001$ \\
\hline Overall & 100 & $5.6 \pm 1.2$ & $62.9 \pm 2.0$ & 0.811 & $<0.001$ \\
\hline
\end{tabular}

RAPS: Rheumatoid Arthritis Pain Scale; CDAI: Clinical Disease Activity Index; CC: Correlation coefficient; SD: Standard deviation; Disease severity as per Clinical Disease Activity Index: remission=0-2.8; mild=2.81-10.0; moderate=10.1-22.0; severe=22.1-76; $p<0.05$ : Significant.

DAS28 and CDAI. Mean values of DAS28, CDAI and RAPS for each category and correlation between the two variables were also assessed. All patients were reassessed at third month follow-up using DAS28 and CDAI while they were also subjected to complete RAPS. Then, the correlation between RAPS change and the change in disease activity was assessed. Cronbach's alpha $(\alpha)$ was calculated for RAPS, DAS28, and CDAI to assess the reliability.

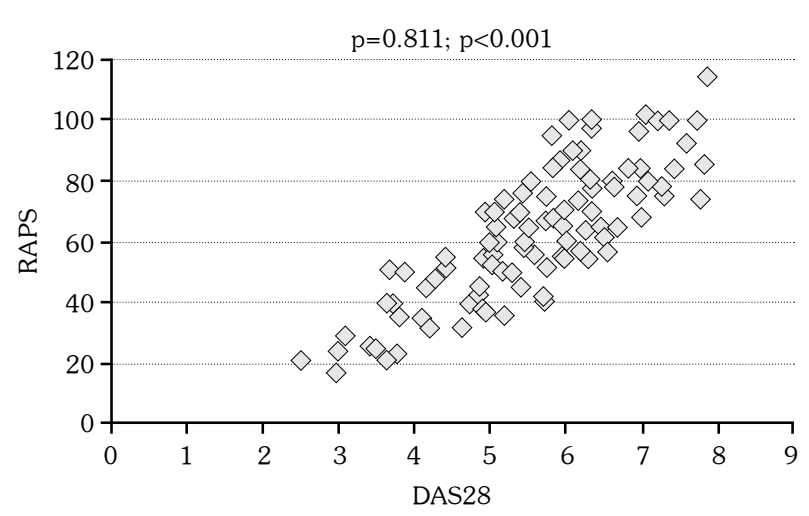

Figure 2. Correlation of RAPS with DAS28.

RAPS: Rheumatoid Arthritis Pain Scale; DAS28: Disease activity score 28.

\section{RESULTS}

In the study group, there were 77 females and 23 males, with a female to male ratio of $3.34: 1$. Mean age of study population was $43.22 \pm 10.4$ years and mean duration of disease was $55.9 \pm 50.6$ months. Rheumatoid factor (RF) positivity was found in 82 patients.

The mean values of various parameters, disease activity indices, and RAPS are shown

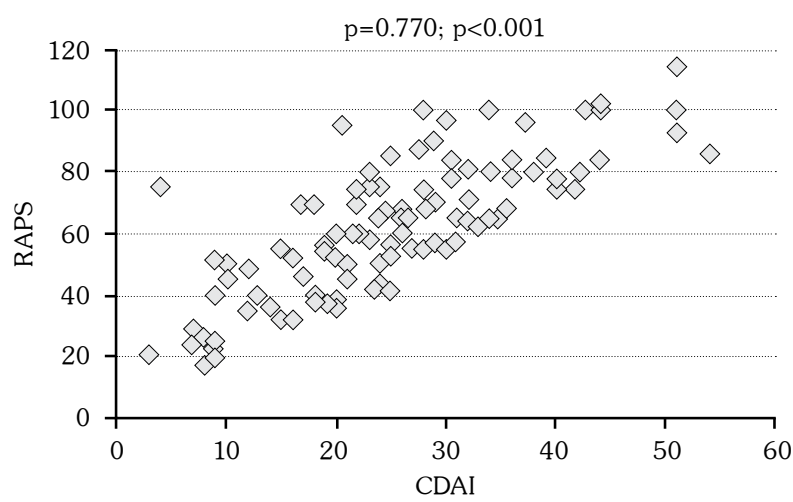

Figure 3. Correlation of RAPS with CDAI.

RAPS: Rheumatoid Arthritis Pain Scale; CDAI: Clinical Disease Activity Index. 
Table 4. Correlation of RAPS with changes in disease activity

\begin{tabular}{|c|c|c|c|c|c|c|}
\hline Mean RAPS change & Disease activity change & & & Disease activity change & & \\
\hline RAPS & DAS28 & $\mathrm{CC}$ & & CDAI & $\mathrm{CC}$ & \\
\hline Mean \pm SD & Mean \pm SD & $p$ & $p$ & Mean \pm SD & $p$ & $p$ \\
\hline$-28.74 \pm 11.54$ & $-1.3 \pm 0.5$ & 0.896 & $<0.001$ & $-11.1 \pm 5.8$ & 0.837 & $<0.001$ \\
\hline
\end{tabular}

RAPS: Rheumatoid Arthritis Pain Scale; DAS28: Disease activity score 28; CC: Correlation coefficient; CDAI: Clinical Disease Activity Index; SD: Standard deviation; $p<0.05$ : Significant.

in Table 1 and Figure 1. RAPS was found to be correlated significantly with DAS28 (Spearman's correlation coefficient $[\rho]=0.811, p<0.001)$ and CDAI $(\rho=0.770, p<0.001)$ (Tables 2, 3 and Figures 2, 3).

Rheumatoid Arthritis Pain Scale was found to be correlated strongly with DAS28 and CDAI in patients who had moderate to severe disease activity (Tables 2, 3). At low disease state, RAPS had fair correlation with DAS28 whereas it was very poorly correlated with CDAI (Tables 2, 3). Correlation of RAPS with DAS28 and CDAI could not be established at remission state due to the inadequate number of subjects (Tables 2, 3).

To evaluate the efficacy for RAPS in term of correlation of its value change with the disease activity change, we re-assessed the RAPS, DAS28 and CDAI at third month follow-up and found a strong positive correlation between RAPS change and changes in DAS28 $(\rho=0.896, p<0.001)$ and CDAI $(\rho=0.837, p<0.001)$ (Table 4; Figures 4, 5).

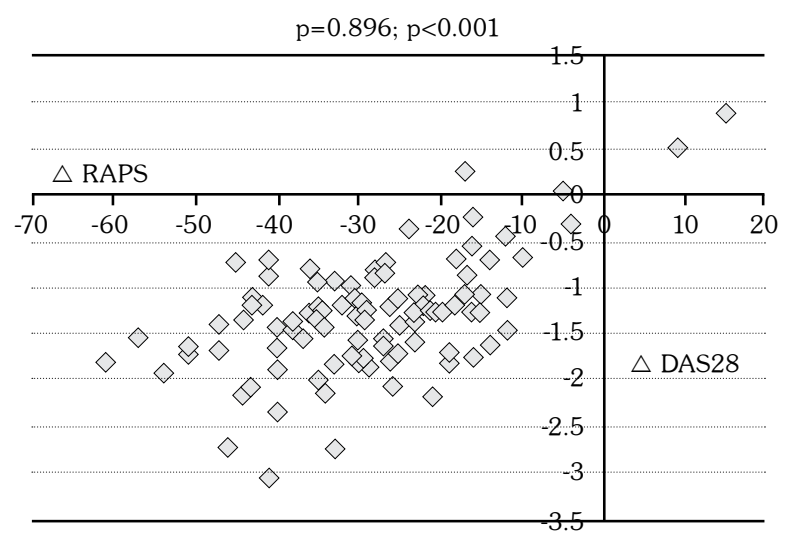

Figure 4. Correlation of RAPS change ( $\triangle$ RAPS on X-axis) with DAS28 change ( $\triangle$ DAS28 on Y-axis).

RAPS: Rheumatoid Arthritis Pain Scale; DAS28: Disease activity score 28.
When RAPS was compared statistically with other core data set measures including TJC, SJC, PGA, EGA, pain VAS, and ESR, it was found to be correlated statistically significantly with coefficients ( $\rho$ ) of $0.753,0.594,0.674$, $0.614,0.641$, and 0.676 , respectively (all $p<0.01$ ) (Table 5).

\section{DISCUSSION}

Pain in RA is the most difficult variable to be quantified as it varies from person to person. It is the major factor for seeking medical attention and determining change in treatment in patients of RA. Pain is a core efficacy set measure for clinical research hence its evaluation becomes necessary. A study by Pincus and Sokka highlighted the importance of pain over joint counts or laboratory parameters in the assessment of disease. ${ }^{7}$ Multiple factors play role in pain experience as a whole like ethnicity, memory, age, religion, and socioeconomic environment.

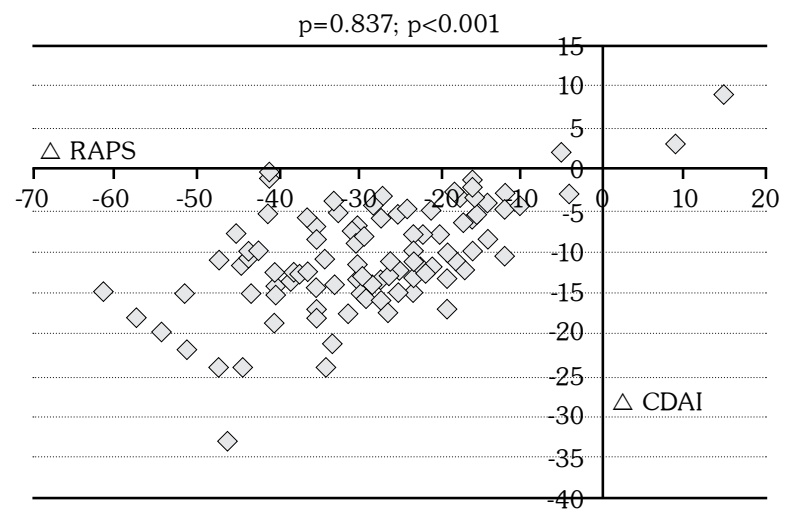

Figure 5. Correlation of RAPS change ( $\triangle$ RAPS on $\mathrm{X}$-axis) with CDAI change ( $\triangle \mathrm{CDAI}$ on Y-axis).

RAPS: Rheumatoid Arthritis Pain Scale; CDAI: Clinical Disease Activity Index. 
Table 5. Correlation of RAPS with various core data set parameters

\begin{tabular}{lcc}
\hline Parameters & Spearman's rho & $p$ \\
\hline Tender joint count & 0.753 & $<0.001$ \\
Swollen joint count & 0.594 & $<0.001$ \\
Patient's global assessment & 0.674 & $<0.001$ \\
Evaluator's global assessment & 0.614 & $<0.001$ \\
Pain visual analog scale & 0.641 & $<0.001$ \\
Erythrocyte sedimentation rate & 0.676 & $<0.001$ \\
\hline $\begin{array}{l}\text { RAPS: Rheumatoid Arthritis Pain Scale; rho: Correlation coefficient; } \\
\text { p<0.05: Significant. }\end{array}$
\end{tabular}

Pain is a complex process in RA and is not always correlated with disease activity particularly when the disease activity or inflammation is settled. In the initial phases of RA, pain results from inflammation, as evidenced by tenderness and swelling of the joints as well as laboratory findings such as increased C-reactive protein and ESR, anemia, or thrombocytosis. Presence of $\mathrm{RF}$ is also consistent with inflammation. As RA progresses, the damaging effect of erosion of cartilage and bone also causes pain. When the inflammatory process is settled, the joint involved may be damaged so that it is mechanically unstable and more likely to undergo degenerative changes that further contribute to pain. ${ }^{8}$

Pain management is one of the components of treatment strategies in RA as effective pain control may provide enhanced compliance of patients to therapy and participation in physical activity. Therefore, pain assessment as well as other objective features need to be considered

Table 6. Rheumatoid Arthritis Pain Scale questionnaire ${ }^{5}$

\begin{tabular}{|c|c|c|}
\hline No & Pain items & Possible range \\
\hline 1 & I would describe my pain as gnawing. & $0-6$ \\
\hline 2 & I would describe my pain as aching. & $0-6$ \\
\hline 3 & I would use the word exhausting to describe my pain & $0-6$ \\
\hline 4 & I would describe my pain as annoying. & $0-6$ \\
\hline 5 & I am in constant pain & $0-6$ \\
\hline 6 & I would describe my pain as rhythmic & $0-6$ \\
\hline 7 & I have swelling of at least one joint. & $0-6$ \\
\hline 8 & I have morning stiffness of one hour or more. & $0-6$ \\
\hline 9 & I have pain on motion of at least one joint. & $0-6$ \\
\hline 10 & I cannot perform all the everyday tasks I normally would because of pain. & $0-6$ \\
\hline 11 & Pain interferes with my sleep. & $0-6$ \\
\hline 12 & I cannot decrease my pain by using methods other than taking extra medication. & $0-6$ \\
\hline 13 & I would describe my pain as burning. & $0-6$ \\
\hline 14 & I find that I guard my joints to reduce pain. & $0-6$ \\
\hline 15 & I brace myself because of the pain. & $0-6$ \\
\hline 16 & My pain is throbbing in nature. & $0-6$ \\
\hline 17 & I would describe my pain as sharp. & $0-6$ \\
\hline 18 & I would say my pain is severe. & $0-6$ \\
\hline 19 & I feel stiffness in my joints after rest. & $0-6$ \\
\hline 20 & My joints feel hot. & $0-6$ \\
\hline 21 & I feel anxious because of pain. & $0-6$ \\
\hline 22 & I would describe my pain as tingling. & $0-6$ \\
\hline 23 & I feel my pain is uncontrollable. & $0-6$ \\
\hline 24 & I feel helpless to control my pain. & $0-6$ \\
\hline
\end{tabular}

Physiological component: Items 7, 8, 9, 19, and 20.

Affective component: Items 3, 4, 14, and 15.

Sensory-discriminative component: Items $1,2,5,6,13,16,17,18$, and 22.

Cognitive component: Items $10,11,12,21,23$, and 24 .

Rheumatoid Arthritis Pain Scale Score=sum of individual scores of 24 items. 
while determining the treatment. To ensure this, a single reliable scoring system is required that assesses pain. However, complete assessment of pain is a difficult task since a very limited number of tools have been designed to date to capture all domains of pain. The existing pain assessing instruments are general assessment measures and are neither specific to RA nor do they reflect the fluctuating and recurrent nature of chronic pain related to this disease. The traditional methods of measurement include simple descriptive scales, modified VASs, numeric rating scales (NRSs), multidimensional scales, and verbal rating scales, such as the McGill Pain Questionnaire (MPQ), which can be generalized to a variety of pain syndromes.

Although pain VAS is the most commonly used tool to assess pain intensity, it does not appear to suffice in current clinical practice where treatment interventions depend upon how well the pain is controlled. Similar to the pain VAS, the pain NRS evaluates only one component of the pain experience such as pain intensity and therefore does not capture the complex and idiosyncratic nature of the RA pain or improvements due to symptom fluctuations.

On the other hand, RAPS (Table 6) is a tool designed specifically for RA encompassing the cognitive appraisals, sensory phenomenon, behavioral disturbances, and physiological factors in a single instrument. ${ }^{5}$ RAPS was developed in 2001 by Anderson ${ }^{5}$ in view of perceiving pain in all dimensions and conferring specificity for RA; however, its use to date has been restricted to certain ethnic groups. This scale is simple to use unlike MPQ, which is full of complex vocabulary and may not be appropriate for patients with low literacy. ${ }^{9}$ RAPS is more comprehensive than pain VAS and may play a complimentary role in capturing multiple domains of pain. ${ }^{10}$

In a study by Kianifard et al. ${ }^{11}$ on Indian patients, RAPS demonstrated modest correlation with DAS28 while no correlation was studied with CDAI. However, in the present study, we found that RAPS showed strong positive correlation with both disease activity measures of DAS28 and CDAI.

Rheumatoid Arthritis Pain Scale also correlated well with the ACR core data measures of ESR, pain VAS, SJC, TJC, PGA, and EGA. According to a study of Anderson et al., ${ }^{2}$ RAPS was shown to correlate with TJC $(\rho=0.5237, p<0.01)$ and pain VAS $(\rho=0.6756, p<0.01)$ whereas another study by Kianifard et al. ${ }^{11}$ demonstrated no correlation between RAPS and pain VAS while showing fair correlation between RAPS and SJC. In our study, we found good correlation between RAPS and baseline disease activity parameters of ESR, pain VAS, SJC, TJC, PGA, and EGA (Table 5).

The reason for the different results in our study might be due to the fact that majority of the patients in our study either presented at advanced stage of the disease (Tables 1,2 ) or due to their more subjective perception of pain.

We also calculated the internal consistency (Cronbach's $\alpha$ ) to measure the reliability of RAPS. The Cronbach's $\alpha$ for RAPS was 0.892 , whereas it was 0.814 and 0.833 for DAS28 and CDAI, respectively, showing significantly higher reliability for RAPS compared to DAS28 and CDAI.

Correlation of RAPS with the changes including improvement or worsening of disease activity was also assessed in our study and RAPS was detected to have strong positive correlation with the changes in disease activity as assessed by DAS28 and CDAI (Table 4). Hence, our study demonstrated that as disease activity scores increased, RAPS score increased and vice versa and treatment brought changes in both disease activity and pain scores.

Thus, in our study, despite excluding joint counts and other physician-derived components, the RAPS has been shown to correlate with joint counts, EGA, ESR measures on an individual basis as well as with other composite measures (e.g. DAS28, CDAI) that contain such items. We also found that RAPS has high reliability, acceptability, feasibility, and sensitivity to detect any improvement or deterioration in RA disease activity. Hence, this instrument can be used in routine clinical care to follow-up patients of RA and to distinguish clinically effective therapies from the ineffective ones. Comprehensive pain assessment using this instrument can lead to more effective pain management and better physical rehabilitation.

The limitations of our study include the single-center design on a relatively small study population. Moreover, although the study group 
was representative of the center's entire RA patient population, the majority of patients had moderate to severe disease activity. Furthermore, fibromyalgia that coexists in $15-20 \%$ of RA patients could have exerted an influence on pain perception. ${ }^{12}$ In addition, pain is highly subjective and its perception varies from individual to individual. Patient issues may be more related to pain whereas physician's main concern is reduction of disease activity.

In conclusion, RAPS had strong positive correlation with disease activity assessment tools as well as with other disease activity parameters. RAPS seems to be a highly reliable and clinically valuable measure to assess pain as well as disease activity comprehensively in RA patients. We suggest further larger studies with longer follow-ups to assess the long-term efficacy of RAPS in the follow-up of RA.

\section{Declaration of conflicting interests}

The authors declared no conflicts of interest with respect to the authorship and/or publication of this article.

\section{Funding}

The authors received no financial support for the research and/or authorship of this article.

\section{REFERENCES}

1. Bichile L. Clinical features and organ involvement in RA. In: Rao URK, Mahendranath KM, Misra R, Handa R, Chaturvedi V, Krishnamurthy V, editors. Manual of Rheumatology. 4th ed. Mumbai: Indian Rheumatology Association; 2014. p. 176-84.

2. Anderson KO, Bradley LA, Turner RA, Agudelo CA,
Pisko EJ. Pain behavior of rheumatoid arthritis patients enrolled in experimental drug trials. Arthritis Care Res 1994;7:64-8.

3. Skevington SM. Psychological aspects of pain in rheumatoid arthritis: a review. Soc Sci Med 1986;23:567-75.

4. Buckelew SP, Parker JC. Coping with arthritis pain. A review of the literature. Arthritis Care Res 1989;2:136-45.

5. Anderson DL. Development of an instrument to measure pain in rheumatoid arthritis: Rheumatoid Arthritis Pain Scale (RAPS). Arthritis Rheum 2001;45:317-23.

6. Arnett FC, Edworthy SM, Bloch DA, McShane DJ, Fries JF, Cooper NS, et al. The American Rheumatism Association 1987 revised criteria for the classification of rheumatoid arthritis. Arthritis Rheum 1988;31:315-24.

7. Pincus T, Sokka T. Quantitative clinical assessment in busy rheumatology settings: the value of short patient questionnaires. J Rheumatol 2008;35:1235-7.

8. Pisetsky DS, McCleane G. Pain in rheumatoid arthritis and osteoarthritis. In: Argoff CE, McCleane G, editors. Pain management secrets. 3rd ed. New York: Elsevier; 2009. p. 170-83.

9. Burckhardt CS, Jones KD. Adult measures of pain: The McGill Pain Questionnaire (MPQ), Rheumatoid Arthritis Pain Scale (RAPS), Short-Form McGill Pain Questionnaire (SF-MPQ), Verbal Descriptive Scale (VDS), Visual Analog Scale (VAS), and West HavenYale Multidisciplinary Pain Inventory (WHYMPI). Arthritis Rheum 2003;49:S96-104.

10. Sim, J and Waterfield, J. Validity, reliability and responsiveness in the assessment of pain. Physiotherapy Theory and Practice 1997;13:23-37.

11. Kianifard T, Kianyfard T, Chopra A. Validation and relevance of Rheumatoid Arthritis Pain Scale (RAPS) in Indian (Asian) patients suffering from rheumatoid arthritis. Clin Rheumatol 2016;35:63-71.

12. Leeb BF, Andel I, Sautner J, Nothnagl T, Rintelen B. The DAS28 in rheumatoid arthritis and fibromyalgia patients. Rheumatology (Oxford) 2004;43:1504-7. 